\title{
F-INVARIANTS OF DIAGONAL HYPERSURFACES
}

\author{
DANIEL JESÚS HERNÁNDEZ
}

\begin{abstract}
In this note, we derive a formula for the $F$-pure threshold of diagonal hypersurfaces over a perfect field of prime characteristic. We also calculate the associated test ideal at the $F$-pure threshold, and give formulas for higher jumping numbers of Fermat hypersurfaces.
\end{abstract}

\section{INTRODUCTION}

Let $R$ be a polynomial ring over a perfect field $\mathbb{L}$ of characteristic $p>0$, and consider a polynomial $f \in R$. Using the Frobenius morphism $R \rightarrow R$ given by $r \mapsto r^{p}$, one may define a family of ideals $\{\boldsymbol{\tau}(\lambda \bullet f) \subseteq R: \lambda>0\}$ called the test ideals of $f$. Test ideals (defined in the context of tight closure) were originally introduced in [HH90], and generalized to pairs in [HY03]. Test ideals vary with respect to $\lambda$ in the following way: they shrink as $\lambda$ increases, and are also stable to the right. We say that a parameter $\lambda$ is an $F$-jumping number of $f$ if $\boldsymbol{\tau}(\lambda \bullet f) \neq \boldsymbol{\tau}((\lambda-\varepsilon) \bullet f)$ for every $0<\varepsilon<\lambda$. We call the smallest $F$-jumping number the $F$-pure threshold of $f$ and denote it by fpt $(f)$. In this article, we consider these invariants when $f$ is diagonal or Fermat. Recall that $f$ is called diagonal if it is an $\mathbb{L}^{*}$-linear combination of $x_{1}^{d_{1}}, \cdots, x_{n}^{d_{n}}$ and Fermat if it is an $\mathbb{L}^{*}$-linear combination of $x_{1}^{d}, \cdots, x_{d}^{d}$.

In Theorem 3.1, we give a formula for the $F$-pure threshold of a diagonal hypersurface as a function of the characteristic. In Theorem 3.3, we give a formula for the first non-trivial test ideal of a diagonal hypersurface. Note that (classical) test ideals of diagonal hypersurfaces were computed by McDermott in [McD01] and [McD03]. In Theorem 3.6, we give conditions for the existence of, and formulas for, higher jumping numbers of Fermat hypersurfaces. For a detailed discussion of our main results, and for examples, see Section 3.

0.1. Acknowledgements. This work is part of the author's Ph.D. thesis at the University of Michigan. I would like to thank Karen Smith for suggesting this problem, as well as Emily Witt, whose observation led to the statement and proof of Theorem 3.1.

\section{Test IdeAls And $F$-PURE THRESholds}

Let $\mathbb{L}$ be a a perfect of characteristic $p>0$, and let $R=\mathbb{L}\left[x_{1}, \cdots, x_{n}\right]$. We will use $\mathfrak{m}$ to denote the ideal $\left(x_{1}, \cdots, x_{n}\right)$. As $\mathbb{L}$ is perfect, we have that $R^{p^{e}}:=\mathbb{L}\left[x_{1}^{p^{e}}, \cdots, x_{n}^{p^{e}}\right]$ is the subring of $\left(p^{e}\right)^{\text {th }}$ powers of $R$. For every ideal $I \subseteq R$, let $I^{\left[p^{e}\right]}$ denote the ideal generated by the set $\left\{g^{p^{e}}: g \in I\right\}$. We call $I^{\left[p^{e}\right]}$ the $e^{\text {th }}$ Frobenius power of $I$.

Definition 1.1. We will use $\mathscr{B}_{e}$ to denote the set of monomials $\left\{\mu: \mu \notin \mathfrak{m}^{\left[p^{e}\right]}\right\}$. The reader may verify that $\mathscr{B}_{e}$ is a free basis for $R$ as an $R^{p^{e}}$-module. If $f \in R$ is a non-zero polynomial and $\mu \in \mathscr{B}_{e}$, we use $\Gamma_{\mu}^{e}(f)$ to denote the element of $R$ such that $f=\sum_{\mu \in \mathscr{B}_{e}} \Gamma_{\mu}^{e}(f)^{p^{e}} \mu$.

The author was partially supported by the National Science Foundation RTG grant number 0502170 at the University of Michigan. 
Remark 1.2. As $R$ is finitely generated and free over $R^{p^{e}}$, it follows that $f^{p^{e}} \in I^{\left[p^{e}\right]}$ if and only if $f \in I$.

Definition 1.3. Let $f \in R$ be a non-zero polynomial. We use $(f)^{\left[\frac{1}{p^{e}}\right]}$ to denote the ideal generated by the set $\left\{\Gamma_{\mu}^{e}(f): \mu \in \mathscr{B}_{e}\right\}$.

Lemma 1.4 follows from [BMS08, Proposition 2.5], though we include a proof for the sake of completeness.

Lemma 1.4. Let $f \in R$. If $I \subseteq R$ is an ideal, then $(f)^{\left[\frac{1}{p^{e}}\right]} \subseteq I$ if and only if $f \in I^{\left[p^{e}\right]}$.

Proof. If $(f)^{\left[\frac{1}{p^{e}}\right]} \subseteq I$, then $f \in\left((f)^{\left[\frac{1}{p^{e}}\right]}\right)^{\left[p^{e}\right]} \subseteq I^{\left[p^{e}\right]}$. Instead, suppose $f \in I^{\left[p^{e}\right]}=\left(a_{1}^{p^{e}}, \cdots, a_{s}^{p^{e}}\right)$.

Then, $f=\sum_{i=1}^{s} g_{i} \cdot a_{i}^{p^{e}}=\sum_{\mu \in \mathscr{B}_{e}}\left(\sum_{i=1}^{s} a_{i} \Gamma_{\mu}^{e}\left(g_{i}\right)\right)^{p^{e}} \mu$. Thus, $\Gamma_{\mu}^{e}(f)=\sum_{i=1}^{s} a_{i} \Gamma_{\mu}^{e}\left(g_{i}\right)$, and we conclude that $(f)^{\left[\frac{1}{p^{e}}\right]} \subseteq I$.

Remark 1.5. Lemma 1.4 shows that $(f)^{\left[\frac{1}{p^{e}}\right]}$ is the unique minimal ideal $I$ such that $f \in I^{\left[p^{e}\right]}$. This shows that $(f)^{\left[\frac{1}{p^{e}}\right]}$ does not depend on the specific choice of basis $\mathscr{B}_{e}$ for $R$ over $R^{p^{e}}$.

Definition 1.6. For every $\lambda \geq 0$, the set $\left\{\left(f^{\left\lceil p^{e} \lambda\right\rceil}\right)^{\left[\frac{1}{p^{e}}\right]}: e \geq 1\right\}$ defines an increasing sequence of ideals [BMS08, Lemma 2.8]. We call the stabilizing ideal the test ideal of $f$ (with respect to the parameter $\lambda$ ), and denote it by $\boldsymbol{\tau}(\lambda \bullet f)$. In other words,

$$
\boldsymbol{\tau}(\lambda \bullet f)=\bigcup_{e \geq 1}\left(f^{\left\lceil p^{e} \lambda\right\rceil}\right)^{\left[\frac{1}{p^{e}}\right]}=\left(f^{\left\lceil p^{e} \lambda\right\rceil}\right)^{\left[\frac{1}{p^{e}}\right]} \text { for all } e \gg 0 .
$$

The following lemma, whose proof we omit, allows us to identify when the test ideal stabilizes in an important special case.

Lemma 1.7. [BMS09, Lemma 2.1] If $\lambda \in \frac{1}{p^{e}} \cdot \mathbb{N}$, then $\boldsymbol{\tau}(\lambda \bullet f)=\left(f^{p^{e} \lambda}\right)^{\left[\frac{1}{p^{e}}\right]}$.

Test ideals form a decreasing sequence of ideals, and are stable to the right [BMS08, Proposition 2.11, Corollary 2.16] . That is, $\boldsymbol{\tau}(\lambda \bullet f) \subseteq \boldsymbol{\tau}\left(\lambda_{\circ} \bullet f\right)$ if $\lambda \geq \lambda_{\circ}$. Additionally, for every $\lambda \geq 0$ there exists $\varepsilon>0$ such that $\boldsymbol{\tau}(\lambda \bullet f)=\boldsymbol{\tau}((\lambda+\delta) \bullet f)$ whenever $0 \leq \delta<\varepsilon$. This behavior motivates the following definition.

Definition 1.8. We say that $\lambda>0$ is an F-jumping number of $f$ if

$$
\boldsymbol{\tau}(\lambda \bullet f) \neq \boldsymbol{\tau}((\lambda-\varepsilon) \bullet f) \text { for all } 0<\varepsilon<\lambda .
$$

By convention, we consider 0 an $F$-jumping number of $f$.

Proposition 1.9. [BMS08, Proposition 2.25] A number $\gamma>1$ is an $F$-jumping number of $f$ if and only if $\gamma-1$ is an $F$-jumping number of $f$.

Let $f$ be a non-zero, non-unit polynomial in $R$, and choose $e \gg 0$ so that $p^{e}>\operatorname{deg} f$. It follows that for every proper ideal $I \subsetneq R$, we have that $f \notin I^{\left[p^{e}\right]}$. This, combined with Lemma 1.4 and Lemma 1.7, shows that $(f)^{\left[\frac{1}{p^{e}}\right]}=\boldsymbol{\tau}\left(\frac{1}{p^{e}} \bullet f\right)$ is not contained in any proper ideal of $R$, and thus must equal $R$. We see that $\boldsymbol{\tau}(\lambda \bullet f)=R$ for $0<\lambda \ll 1$, and so the smallest non-zero $F$-jumping number of $f$ is the minimal parameter $\lambda$ such that $\boldsymbol{\tau}(\lambda \bullet f) \neq R$. This jumping number is of particular interest, and is called the $F$-pure threshold of $f$. 
Definition 1.10. We call fpt $(f):=\sup \left\{\lambda \in \mathbb{R}_{\geq 0}: \boldsymbol{\tau}(\lambda \bullet f)=R\right\}$ the F-pure threshold of $f$, and we call $\mathbf{f p t}_{\mathfrak{m}}(f):=\sup \left\{\lambda \in \mathbb{R}_{\geq 0}: \boldsymbol{\tau}(\lambda \bullet \bar{f})_{\mathfrak{m}}=R_{\mathfrak{m}}\right\}$ the $F$-pure threshold of $f$ at $\mathfrak{m}$.

In our computations, we will use the following well known description of $\mathbf{f p t}_{\mathfrak{m}}(f)$.

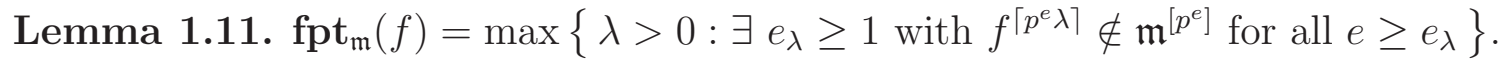

Proof. Comparing with Definition 1.10, we see it suffices to show $\boldsymbol{\tau}(\lambda \bullet f)_{\mathfrak{m}}=R_{\mathfrak{m}}$ if and only if there exists $e_{\lambda} \geq 1$ with $f^{\left[p^{e}\right\rceil} \notin \mathfrak{m}^{\left[p^{e}\right]}$ for all $e \geq e_{\lambda}$. By definition, there exists $e_{\lambda}$ such that $\boldsymbol{\tau}(\lambda \bullet f)=\left(f^{\left\lceil p^{e} \lambda\right]}\right)^{\left[\frac{1}{p^{e}}\right]}$ for all $e \geq e_{\lambda}$. For such an $e, \boldsymbol{\tau}(\lambda \bullet f)_{\mathfrak{m}}=R_{\mathfrak{m}}$ if and only

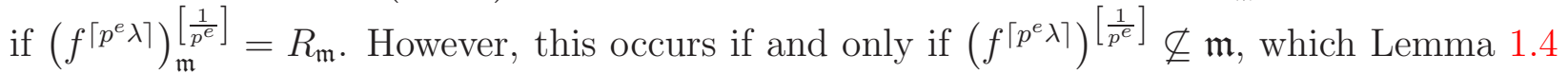
shows happens if and only if $f^{\left\lceil p^{e} \lambda\right\rceil} \notin \mathfrak{m}^{\left[p^{e}\right]}$.

\section{SOME REMARKS ON BASE $p$ EXPANSIONS}

Definition 2.1. Let $\alpha \in(0,1]$, and let $p$ be a prime number. Let $\alpha^{(d)}$ be the unique integer in $[0, p-1]$ such that $\alpha=\sum_{d \geq 1} \frac{\alpha^{(d)}}{p^{d}}$ and such that $\alpha^{(d)} \neq 0$ is not eventually zero as a function of $d$. We call $\alpha^{(d)}$ the $d^{\text {th }}$ digit of the non-terminating base $p$ expansion of $\alpha$. We adopt the convention that $\alpha^{(0)}=0^{(d)}=0$.

Example 2.2. If $\alpha=\frac{1}{p}=\frac{0}{p}+\sum_{e \geq 2}$, we see that $\alpha^{(1)}=0$ and $\alpha^{(e)}=p-1$ for all $e \geq 1$.

Definition 2.3. If $\lambda \neq 0$, we call $\langle\lambda\rangle_{e}:=\sum_{d=1}^{e} \frac{\lambda^{(d)}}{p^{d}}$ the $e^{\text {th }}$ truncation of $\lambda$ (in base $p$ ).

Lemma 2.4. If $\lambda \in[0,1]$, then $\left\lceil p^{e} \lambda\right\rceil=p^{e}\langle\lambda\rangle_{e}+1$. Furthermore, if $\alpha \in[0,1] \cap \frac{1}{p^{e}} \cdot \mathbb{N}$ and $\lambda>\alpha$, then $\langle\lambda\rangle_{e} \geq \alpha$.

Proof. As $p^{e} \lambda=p^{e}\langle\lambda\rangle_{e}+p^{e} \cdot \sum_{d>e} \frac{\lambda^{(d)}}{p^{d}}$, the first claim follows from the observation that $0<\sum_{d>e} \frac{\lambda^{(d)}}{p^{d}} \leq \frac{1}{p^{e}}$. We also see that $\frac{1}{p^{e}}+\langle\lambda\rangle_{e} \geq \lambda>\alpha$, so

$$
1+p^{e}\langle\lambda\rangle_{e}>p^{e} \alpha
$$

By hypothesis, both sides of (1) are integers, and we conclude that $p^{e}\langle\lambda\rangle_{e} \geq p^{e} \alpha$.

Definition 2.5. Let $\left(\lambda_{1}, \cdots, \lambda_{n}\right) \in[0,1]^{n}$, and let $p$ be a prime number. We say the $e^{\text {th }}$ digits of $\lambda_{1}, \cdots, \lambda_{n}$ add without carrying (in base $p$ ) if $\lambda_{1}^{(e)}+\cdots+\lambda_{n}^{(e)} \leq p-1$, and we say that $\lambda_{1}, \cdots, \lambda_{n}$ add without carrying if all of their digits add without carrying. We say natural numbers $k_{1}, \cdots, k_{n}$ add without carrying (in base $p$ ) if the obvious condition holds.

Remark 2.6. If $\lambda_{1}, \cdots, \lambda_{n}$ add without carrying (in base $p$ ) and $\lambda:=\sum_{i=1}^{n} \lambda_{i} \leq 1$, then $\lambda^{(e)}=\lambda_{1}^{(e)}+\cdots+\lambda_{n}^{(e)}$ for all $e \geq 1$.

The notion of adding without carrying is relevant in light of the following classical result.

Lemma 2.7. [Dic02, Luc78] Let $\boldsymbol{k}=\left(k_{1}, \cdots k_{n}\right) \in \mathbb{N}^{n}$ and set $N=|\boldsymbol{k}|=\sum k_{i}$. Then $\left(\begin{array}{l}N \\ k\end{array}\right):=\frac{N !}{k_{1} ! \cdots k_{n} !} \not \equiv 0 \bmod p$ if and only if $k_{1}, \cdots, k_{n}$ add without carrying (in base $p$ ). 


\section{Discussion of the MAin RESUlts}

3.1. $F$-pure theshholds of diagonal hypersurfaces. In our first result, we give a formula the $F$-pure threshold of a diagonal hypersurface.

Theorem 3.1. Let $L$ be the supremum over all $N$ such that the $e^{\text {th }}$ digits of $\frac{1}{d_{1}}, \cdots, \frac{1}{d_{n}}$ add without carrying for all $0 \leq e \leq N$. If $f$ is a $\mathbb{L}^{*}$-linear combination of $x_{1}^{d_{1}}, \cdots, x_{n}^{d_{n}}$, then

$$
\mathbf{f p t}_{\mathfrak{m}}(f)= \begin{cases}\frac{1}{d_{1}}+\cdots+\frac{1}{d_{n}} & \text { if } L=\infty \\ \left\langle\frac{1}{d_{1}}\right\rangle_{L}+\cdots+\left\langle\frac{1}{d_{n}}\right\rangle_{L}+\frac{1}{p^{L}} & \text { if } L<\infty\end{cases}
$$

Formulas for the $F$-pure threshold of $x^{2}+y^{3}$ and $x^{2}+y^{7}$ are given in [MTW05, Example 4.3 and 4.4]. At first glance, these formulas appear to be quite different from those in Theorem 3.1 above. Below, we show how Theorem 3.1 may be used to recover these formulas.

Example 3.2. We adopt decimal notation for base $p$ expansions. For example, if $a, b$ are integers with $0 \leq a, b \leq p-1$, then $\overline{a b}$ (base $p$ ) will denote the unique number $\lambda$ with the property that $\lambda^{(e)}=a$ for $e$ odd and $\lambda^{(e)}=b$ for $e$ even. Let $f$ be a $\mathbb{L}^{*}$-linear combination of $x^{2}$ and $y^{3}$. If $p=3$, then

$$
\frac{1}{2}=. \overline{1} \text { (base } 3 \text { ) and } \frac{1}{3}=.1=.0 \overline{2} \text { (base } 3 \text { ). }
$$

We see that carrying is required to add the second digits of $\frac{1}{2}$ and $\frac{1}{3}$ (but not the first),

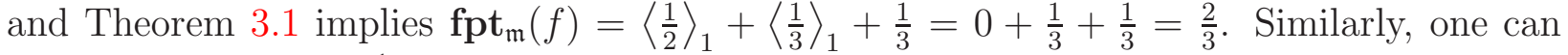
show that $\mathbf{f p t}_{\mathfrak{m}}(f)=\frac{1}{2}$ if $p=2$. If $p=6 \omega+1$ for some $\omega \geq 1$, then

$$
\frac{1}{2}=. \overline{3 \omega}(\text { base } p) \text { and } \frac{1}{3}=. \overline{2 \omega}(\text { base } p) .
$$

We notice that $\frac{1}{2}$ and $\frac{1}{3}$ add without carrying (in base $p$ ), and Theorem 3.1 implies $\mathbf{f p t}_{\mathfrak{m}}(f)=\frac{1}{2}+\frac{1}{3}=\frac{5}{6}$. Finally, if $p=6 \omega+5$ for some $\omega \geq 0$, then

$$
\frac{1}{2}=. \overline{3 \omega+2}(\text { base } p) \text { and } \frac{1}{3}=. \overline{2 \omega+1} 4 \omega+3(\text { base } p) .
$$

Once more, we see that carrying is needed to add the second digits of $\frac{1}{2}$ and $\frac{1}{3}$, (but not the first), and Theorem 3.1 implies

$$
\mathbf{f p t}_{\mathfrak{m}}(f)=\left\langle\frac{1}{2}\right\rangle_{1}+\left\langle\frac{1}{3}\right\rangle_{1}+\frac{1}{p}=\frac{3 \omega+2}{p}+\frac{2 \omega+1}{p}+\frac{1}{p}=\frac{5 \omega+4}{p} .
$$

The reader may verify that $\frac{5 \omega+4}{p}+\frac{1}{6 p}=\frac{5}{6}$, so we may rewrite (2) as $\mathbf{f p t}_{\mathfrak{m}}(f)=\frac{5}{6}-\frac{1}{6 p}$. Thus, we recover the following formula from [MTW05, Example 4.3]:

$$
\mathbf{f p t}_{\mathfrak{m}}\left(x^{2}+y^{3}\right)= \begin{cases}1 / 2 & \text { if } p=2 \\ 2 / 3 & \text { if } p=3 \\ 5 / 6 & \text { if } p \equiv 1 \bmod 6 \\ \frac{5}{6}-\frac{1}{6 p} & \text { if } p \equiv 5 \bmod 6\end{cases}
$$


3.2. A computation of the first non-trivial test ideal. Our second theorem computes the value of the test ideal at the $F$-pure threshold.

Theorem 3.3. If $f$ is a $\mathbb{L}^{*}$-linear combination of $x_{1}^{d_{1}}, \cdots, x_{n}^{d_{n}}$, then

$$
\boldsymbol{\tau}\left(\mathbf{f p t}_{\mathfrak{m}}(f) \bullet f\right)= \begin{cases}(f) & \text { if } \mathbf{f p t}_{\mathfrak{m}}(f)=1 \\ \mathfrak{m} & \text { if } \mathbf{f p t}_{\mathfrak{m}}(f)=\frac{1}{d_{1}}+\cdots+\frac{1}{d_{n}} \\ \mathfrak{m} & \text { if } \mathbf{f p t}_{\mathfrak{m}}(f)<\min \left\{1, \sum_{i=1}^{n} \frac{1}{d_{i}}\right\} \text { and } p>\max \left\{d_{1}, \cdots, d_{n}\right\} .\end{cases}
$$

Remark 3.4. Note that $\boldsymbol{\tau}\left(\mathbf{f p t}_{\mathfrak{m}}(f) \bullet f\right)$ need not equal $\mathfrak{m}$ if $\mathbf{f p t}_{\mathfrak{m}}(f)<\min \left\{1, \sum_{i=1}^{n} \frac{1}{d_{i}}\right\}$ and $p$ is less than or equal to some exponent [MY09, Proposition 4.2].

3.3. On (higher) F-jumping numbers of Fermat hypersurfaces. Our final result computes higher jumping numbers of the degree $d$ Fermat hypersurface. By Proposition 1.9, it suffices to only consider those jumping numbers contained in $(0,1]$. Theorem 3.1 takes the following simple form when $f$ is the degree $d$ Fermat hypersurface.

Corollary 3.5. If $f$ is a $\mathbb{L}^{*}$-linear combination of $x_{1}^{d}, \cdots, x_{d}^{d}$, then

$$
\mathbf{f p t}_{\mathfrak{m}}(f)= \begin{cases}\frac{1}{p^{l}} & \text { if } p^{l} \leq d<p^{l+1} \text { for some } l \geq 1 \\ 1-\frac{a-1}{p} & \text { if } 0<d<p \text { and } p \equiv a \bmod d \text { with } 1 \leq a<d\end{cases}
$$

Theorem 3.6. Suppose that $p>d$ and write $p=d \cdot \omega+a$ for some $\omega \geq 1$ and $1 \leq a<d$. If $f$ is a $\mathbb{L}^{*}$-linear combination of $x_{1}^{d_{1}}, \cdots, x_{n}^{d_{n}}$ and $a=1$, Corollary 3.5 implies that $\mathbf{f p t}_{\mathfrak{m}}(f)=1$. We now assume $a \geq 2$.

(1) If $p<a(d-1)$, then $\mathbf{f p t}_{\mathfrak{m}}(f)<\frac{(d+1) \cdot \omega+\lceil 2 a / d\rceil}{p} \leq 1$ are $F$-jumping numbers in $(0,1]$.

(2) If $p>a(d-1)$, then $\mathbf{f p t}_{\mathfrak{m}}(f)<1$ are the only $F$-jumping numbers in $(0,1]$.

Remark 3.7. As $a$ is strictly less than $d$, Theorem 3.6 implies that $\mathbf{f p t}_{\mathfrak{m}}(f)$ and 1 are the only jumping numbers of $f$ in $(0,1]$ if $p>(d-1)^{2}$.

Example 3.8. Suppose that $d=4$, and $p=7$. Then $\omega=1, a=3$, and $p<a(d-1)$. We see that $(d+1) \cdot \omega+\lceil 2 a / d\rceil=5+\lceil 6 / 4\rceil=7=p$. In this case, Theorem 3.6 provides no new information.

Example 3.9. Instead, let $d=6$ and $p=11$, so that $\omega=1, a=5$, and $p<a(d-1)$. We see that $(d+1) \cdot \omega+\lceil 2 a / d\rceil=7+\lceil 10 / 5\rceil=9$. Corollary 3.5 and Theorem 3.6 then imply $\mathbf{f p t}_{\mathfrak{m}}(f)=1-\frac{a-1}{p}=\frac{7}{11}, \frac{(d+1) \cdot \omega+\lceil 2 a / d\rceil}{p}=\frac{9}{11}$, and 1 are $F$-jumping numbers of $f$ contained in $(0,1]$. The reader may verify that these are all of the $F$-jumping numbers of $f$ in $(0,1]$

\section{F-PURE THRESHOLDS OF DIAGONAL HYPERSURFACES}

Notation 4.1. Set $\delta_{i}=\frac{1}{d_{i}}, \boldsymbol{\delta}:=\left(\delta_{1}, \cdots, \delta_{n}\right)$, and $\langle\boldsymbol{\delta}\rangle_{e}:=\left(\left\langle\delta_{1}\right\rangle_{e}, \cdots,\left\langle\delta_{n}\right\rangle_{e}\right)$. As in the statement of Theorem 3.1, $L=\sup \left\{N: \delta_{1}^{(e)}+\cdots+\delta_{n}^{(e)} \leq p-1\right.$ for all $\left.0 \leq e \leq N\right\}$.

$\mathbf{D}$ will denote the diagonal matrix whose $i^{\text {th }}$ diagonal entry is $d_{i}$, and we set $\boldsymbol{\Delta}:=\mathbf{D}^{-1}$. Note that $\boldsymbol{\Delta}$ is also diagonal, with the $i^{\text {th }}$ diagonal entry being $\delta_{i}$. Throughout this chapter, 
we assume that $f$ is a $\mathbb{L}^{*}$-linear combination of $x_{1}^{d_{1}}, \cdots, x_{n}^{d_{n}}$, and write $f=u_{1} x^{d_{1}}+\cdots+u_{n} x^{d_{n}}$. Using multi-index notation,

$$
f^{N}=\sum_{|\boldsymbol{k}|=N}\left(\begin{array}{l}
N \\
\boldsymbol{k}
\end{array}\right) \boldsymbol{u}^{\boldsymbol{k}} \boldsymbol{x}^{\mathrm{D} \boldsymbol{k}} .
$$

If $\boldsymbol{\lambda} \in \mathbb{R}^{n}$, we use $|\boldsymbol{\lambda}|$ to denote the coordinate sum $\lambda_{1}+\cdots+\lambda_{n}$. When considering elements of $\mathbb{R}^{n}, \preccurlyeq\left(\right.$ and $\prec$ ) will denote component-wise (strict) equality. Finally, $\left\{\mathbf{v}_{1}, \cdots, \mathbf{v}_{n}\right\}$ denotes the standard basis of $\mathbb{R}^{n}$, and $\mathbf{1}_{n}:=(1, \cdots, 1)$.

Though the first part of Theorem 3.1 follows directly from a more general statement from [Her11], we have included a proof below in this simple case.

Theorem 3.1: Part I. If $L=\infty$, then $\mathbf{f p t}_{\mathfrak{m}}(f)=|\boldsymbol{\delta}|$.

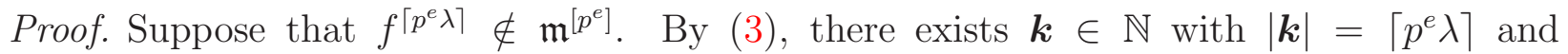
$\mathbf{D} \boldsymbol{k} \prec\left(p^{e}-1\right) \cdot \mathbf{1}_{n}$, so that $\boldsymbol{k} \prec\left(p^{e}-1\right) \cdot \boldsymbol{\delta}$. Thus, $p^{e} \lambda \leq\left\lceil p^{e} \lambda\right\rceil=|\boldsymbol{k}|<\left(p^{e}-1\right)|\boldsymbol{\delta}|$, and so $\lambda<|\boldsymbol{\delta}|$. It follows from Lemma 1.11 that $\mathbf{f p t}_{\mathfrak{m}}(f) \leq|\boldsymbol{\delta}|$.

As $L=\infty$, the entries of $\boldsymbol{\delta}$ add without carrying (in base $p$ ), and it follows that the entries of $p^{e}\langle\boldsymbol{\delta}\rangle_{e}$ add without carrying for all $e \geq 1$. By Lemma 2.7, $\left(\begin{array}{c}p^{e}\left|\langle\boldsymbol{\delta}\rangle_{e}\right| \\ p^{e}\langle\boldsymbol{\delta}\rangle_{e}\end{array}\right) \neq 0 \bmod p$, and as $\mathbf{D}\langle\boldsymbol{\delta}\rangle_{e} \prec \mathbf{D} \boldsymbol{\delta}=\mathbf{1}_{m}$, it follows that monomal $\boldsymbol{x}^{\mathbf{D}\langle\delta\rangle_{e}} \notin \mathfrak{m}^{\left[p^{e}\right]}$.

Combining this with (3), shows that $f^{p^{e} \mid\langle\boldsymbol{\delta}\rangle} \mid \notin \mathfrak{m}^{\left[p^{e}\right]}$, and Remark 1.2 then shows that $f^{p^{d}\langle\boldsymbol{\delta}\rangle_{e} \notin \mathfrak{m}\left[p^{d}\right]}$ for all $d \geq e$. Lemma 1.11 shows that $\mathbf{f p t}_{\mathfrak{m}}(f) \geq\left|\langle\boldsymbol{\delta}\rangle_{e}\right|$ for all $e$, and the claim follows by letting $e \rightarrow \infty$.

Theorem 3.1: Part II. If $L<\infty$, then $\mathbf{f p t}_{\mathfrak{m}}(f)=\left|\langle\boldsymbol{\delta}\rangle_{L}\right|+\frac{1}{p^{L}}$.

Proof. The estimate for $F$-pure thresholds given in [Her11, Main Theorem] implies that

$$
\mathbf{f p t}_{\mathfrak{m}}(f) \geq\langle\boldsymbol{\delta}\rangle_{L}+\frac{1}{p^{L}}
$$

If the inequality in (4) is strict, then Lemma 1.11 implies there exists $e \geq L$ such that

$$
\left(f^{p^{L}\left|\langle\boldsymbol{\delta}\rangle_{L}\right|+1}\right)^{p^{e-L}}=f^{p^{e}\left|\langle\boldsymbol{\delta}\rangle_{L}\right|+p^{e-L}} \notin \mathfrak{m}^{\left[p^{e}\right]}
$$

By Remark 1.2, it follows that $f^{p^{L}\left|\langle\boldsymbol{\delta}\rangle_{L}\right|+1} \notin \mathfrak{m}^{\left[p^{L}\right]}$. Applying (3) shows there exists $\boldsymbol{k} \in \mathbb{N}^{n}$ such that $|\boldsymbol{k}|=p^{L}\left|\langle\boldsymbol{\delta}\rangle_{L}\right|+1$ and $\boldsymbol{x}^{\mathbf{D} \boldsymbol{k}} \notin \mathfrak{m}{ }^{\left[p^{L}\right]}$. This last condition implies that $\frac{1}{p^{L}} \cdot \boldsymbol{k} \prec \boldsymbol{\delta}$, and applying Lemma 2.4 then shows $\frac{1}{p^{L}} \cdot \boldsymbol{k} \preccurlyeq\langle\boldsymbol{\delta}\rangle_{L}$. Thus, $\left|\langle\boldsymbol{\delta}\rangle_{L}\right|+\frac{1}{p^{L}}=\frac{1}{p^{L}} \cdot \boldsymbol{k} \leq\left|\langle\boldsymbol{\delta}\rangle_{L}\right|$, a contradiction. We conclude that equality holds in (4), and so we are done.

\section{Test ideAls of Diagonal hyPERSURFACES}

We now prove Theorem 3.3 in three parts. As before, we assume $f$ is a $\mathbb{L}^{*}$-linear combination of $x_{1}^{d_{1}}, \cdots, x_{n}^{d_{n}}: f=\sum_{i=1}^{n} u_{i} x_{i}^{d_{i}}$. We also continue to adopt Notation 4.1.

Theorem 3.3: Part I. If $\mathbf{f p t}_{\mathfrak{m}}(f)=1$, then $\boldsymbol{\tau}\left(\mathbf{f p t}_{\mathfrak{m}}(f) \bullet f\right)=(f)$.

Proof. Note that $f^{p^{e}}=f^{p^{e}} \cdot 1$, and that $1 \in \mathscr{B}_{e}$. This, $\Gamma_{1}^{e}\left(f^{p}\right)=f$ while $\Gamma_{\mu}^{e}\left(f^{p}\right)=0$ for all $1 \neq \mu \in \mathscr{B}_{e}$. It follows from this, and Lemma 1.7, that $(f)=\left(f^{p^{e}}\right)^{\left[\frac{1}{p^{e}}\right]}=\boldsymbol{\tau}(1 \bullet f)$. 
To prove the remaining parts of Theorem 3.3, we will need Corollary 5.4 below.

Lemma 5.1. The natural number $d_{i}\left(p^{e}\left\langle\delta_{i}\right\rangle_{e}+1-p^{e} \delta_{i}\right)$ is less than $d_{i}$. In particular, if $d_{i} \leq p^{e}$, then $\mathbf{D}\left(p^{e}\langle\boldsymbol{\delta}\rangle_{e}+\left(1-p^{e} \delta_{i}\right) \cdot \mathbf{v}_{i}\right) \prec p^{e} \cdot \mathbf{1}_{n}$.

Proof. As $\left\langle\frac{1}{d}\right\rangle_{e}<\frac{1}{d}$, it follows that $d_{i}\left(p^{e}\left\langle\delta_{i}\right\rangle_{e}+1-p^{e} \delta_{i}\right)<d_{i}\left(p^{e} \delta_{i}+1-p^{e} \delta_{i}\right)=d_{i}$.

In the proof of Lemma 5.2, we use $\bullet$ to denote the standard dot product on $\mathbb{R}^{n}$.

Lemma 5.2. Suppose that $d_{i}<p^{e}$ and that $d_{i}$ is not a power of $p$. By Lemma 5.1, $\mu_{i}:=\boldsymbol{x}^{\mathbf{D}\left(p^{e}\langle\boldsymbol{\delta}\rangle_{e}+\left(1-p^{e} \delta_{i}\right) \cdot \mathbf{v}_{i}\right)} \in \mathscr{B}_{e}$ and $\Gamma_{\mu_{i}}^{e}\left(f^{p^{e}\left|\langle\boldsymbol{\delta}\rangle_{e}\right|+1}\right)=\left(\left(\begin{array}{c}\left|p^{e}\langle\boldsymbol{\delta}\rangle_{e}\right|+1 \\ p^{e}\langle\boldsymbol{\delta}\rangle_{e}+\mathbf{v}_{i}\end{array}\right) \boldsymbol{u}^{p^{e}\langle\boldsymbol{\delta}\rangle_{e}+\mathbf{v}_{i}}\right)^{1 / p^{e}} \cdot x_{i}$.

Proof. To calculate $\Gamma_{\mu_{i}}^{e}\left(f^{p^{e}\left|\langle\boldsymbol{\delta}\rangle_{e}\right|+1}\right)$, we must determine which (possibly) supporting monomials of $f^{p^{e}\left|\langle\boldsymbol{\delta}\rangle_{e}\right|+1}$ are $R^{p^{e}}$ multiples of $\mu_{i}$. A monomial satisfying this condition is of form $\boldsymbol{x}^{\mathbf{D} \boldsymbol{k}}$ for some $\boldsymbol{k} \in \mathbb{N}$ with $|\boldsymbol{k}|=p^{e}\left|\langle\boldsymbol{\delta}\rangle_{e}\right|+1$ such that $\mathbf{D} \boldsymbol{k}=p^{e} \boldsymbol{a}+\mathbf{D}\left(p^{e}\langle\boldsymbol{\delta}\rangle_{e}+\left(1-p^{e} \delta_{i}\right) \cdot \mathbf{v}_{i}\right)$ for some vector $\boldsymbol{a}$. Applying $\boldsymbol{\Delta}=\mathbf{D}^{-1}$ then shows that

$$
\boldsymbol{k}=p^{e} \boldsymbol{\Delta} \boldsymbol{a}+p^{e}\langle\boldsymbol{\delta}\rangle_{e}+\left(1-p^{e} \delta_{i}\right) \cdot \mathbf{v}_{i} .
$$

If $a_{i}=0$, (6) shows that $k_{i}=p^{e}\left\langle\delta_{i}\right\rangle_{e}+1-p^{e} \delta_{i}$, so that $p^{e} \delta_{i} \in \mathbb{N}$, which contradicts the assumption that $d_{i}$ is not a power of $p$. Thus, $a_{i} \geq 1$. By summing the equation appearing in (6), we see that $p^{e}\left|\langle\boldsymbol{\delta}\rangle_{e}\right|+1=|\boldsymbol{k}|=p^{e} \boldsymbol{\delta} \bullet \boldsymbol{a}+p^{e}\left|\langle\boldsymbol{\delta}\rangle_{e}\right|+1-p^{e} \delta_{i}$, and so

$$
\boldsymbol{\delta} \bullet\left(\boldsymbol{a}-\mathbf{v}_{i}\right)=0 .
$$

As $a_{i} \geq 1, \boldsymbol{a}-\mathbf{v}_{i} \succcurlyeq \mathbf{0}$, and as the entries of $\boldsymbol{\delta}$ are non-zero, follows from (6) that $\boldsymbol{a}=\mathbf{v}_{i}$. Substituting this into (6) shows that the only (possibly) supporting monomial of $f^{p^{e}}\left|\langle\boldsymbol{\delta}\rangle_{e}\right|+1$ that is an $R^{p^{e}}$-multiple of $\mu_{i}$ is $\boldsymbol{x}^{\mathbf{D}\left(p^{e}\langle\boldsymbol{\delta}\rangle_{e}+\mathbf{v}_{i}\right)}=\boldsymbol{x}^{\mathbf{D}\left(p^{e}\langle\boldsymbol{\delta}\rangle_{e}+\left(1-p^{e} \delta_{i}\right) \mathbf{v}_{i}\right)} \cdot \boldsymbol{x}^{\mathbf{D} p^{e} \delta_{i}}=\mu_{i} \cdot x_{i}^{p^{e}}$.

Lemma 5.3. If $\sum_{i=1}^{n} \delta_{i}^{(e)} \leq p-2$ and $\left(\begin{array}{c}\left|p^{e}\langle\boldsymbol{\delta}\rangle_{e}\right| \\ p^{e}\langle\boldsymbol{\delta}\rangle_{e}\end{array}\right) \neq 0 \bmod p$, then $\left(\begin{array}{c}\left|p^{e}\langle\boldsymbol{\delta}\rangle_{e}\right|+1 \\ p^{e}\langle\boldsymbol{\delta}\rangle_{e}+\mathbf{v}_{i}\end{array}\right) \neq 0 \bmod p$.

Proof. Note that $\delta_{i}^{(e)} \leq \sum_{i=1}^{n} \delta_{i}^{(e)} \leq p-2$, which implies that both $p^{e}\left\langle\delta_{i}\right\rangle_{e}+1 \equiv \delta_{i}^{(e)}+1$ and $p^{e}\left|\langle\boldsymbol{\delta}\rangle_{e}\right|+1 \equiv \sum_{i=1}^{n} \delta_{i}^{(e)}$ are non-zero $\bmod p$. The claim by reducing the equality $\left(p^{e}\left\langle\delta_{i}\right\rangle_{e}+1\right) \cdot\left(\begin{array}{c}\left|p^{e}\langle\boldsymbol{\delta}\rangle_{e}\right|+1 \\ p^{e}\langle\boldsymbol{\delta}\rangle_{e}+\mathrm{v}_{i}\end{array}\right)=\left(\begin{array}{c}\left|p^{e}\langle\boldsymbol{\delta}\rangle_{e}\right| \\ p^{e}\langle\boldsymbol{\delta}\rangle_{e}\end{array}\right) \cdot\left(\left|p^{e}\langle\boldsymbol{\delta}\rangle_{e}\right|+1\right) \bmod p$.

Corollary 5.4. Suppose that $d_{i}<p^{e}$ and is not a power of $p$. If $\sum_{i=1}^{n} \delta_{i}^{(e)} \leq p-2$ and $\left(\begin{array}{c}\left|p^{e}\langle\boldsymbol{\delta}\rangle_{e}\right| \\ p^{e}\langle\boldsymbol{\delta}\rangle_{e}\end{array}\right) \neq 0 \bmod p$, then $x_{i} \in\left(f^{p^{e}\left|\langle\boldsymbol{\delta}\rangle_{e}\right|+1}\right)^{\left[\frac{1}{p^{e}}\right]}$.

Proof. This follows immediately from Lemmas 5.2 and 5.3.

Theorem 3.3: Part II. If $\mathbf{f p t}_{\mathfrak{m}}(f)=|\boldsymbol{\delta}|<1$, then $\boldsymbol{\tau}\left(\mathbf{f p t}_{\mathfrak{m}}(f) \bullet f\right)=\mathfrak{m}$.

Proof. By Theorem 3.1, the entries of $\boldsymbol{\delta}$ add without carrying (in base $p$ ), so that no $d_{i}$ is a $p^{\text {th }}$ power (for else carrying would be necessary) and $\left(\begin{array}{c}\left|p^{e}\langle\boldsymbol{\delta}\rangle_{e}\right| \\ p^{e}\langle\boldsymbol{\delta}\rangle_{e}\end{array}\right) \neq 0 \bmod p$ for all $e \geq 1$, by Lemma 2.7. As no $d_{i}$ is a $p^{\text {th }}$ power and $|\boldsymbol{\delta}|<1$, the denominator of $|\boldsymbol{\delta}|$ is also not a $p^{\text {th }}$ power, and applying Remark 2.6 shows $\sum_{i=1}^{n} \delta_{i}^{(e)}=|\boldsymbol{\delta}|^{(e)}<p-1$ for infinitely many $e$. Choose such an $e$ so that additionally every $d_{i}$ is less than $p^{e}$ and

$$
\boldsymbol{\tau}(|\boldsymbol{\delta}| \bullet f)=\left(f^{\left\lceil p^{e}|\boldsymbol{\delta}|\right\rceil}\right)_{7}^{\left[\frac{1}{p^{e}}\right]}=\left(f^{p^{e}\left|\langle\boldsymbol{\delta}\rangle_{e}\right|+1}\right)^{\left[\frac{1}{p^{e}}\right]},
$$


where we have used Lemma 2.4 to obtain the equality $\left\lceil p^{e}|\boldsymbol{\delta}|\right\rceil=p^{e}\langle|\boldsymbol{\delta}|\rangle_{e}+1=p^{e}\left|\langle\boldsymbol{\delta}\rangle_{e}\right|+1$. Applying Corollary 5.4 then shows $\left(x_{1}, \cdots, x_{n}\right) \subseteq\left(f^{p^{e}\left|\langle\boldsymbol{\delta}\rangle_{e}\right|+1}\right)^{\left[\frac{1}{p^{e}}\right]}=\boldsymbol{\tau}(|\boldsymbol{\delta}| \bullet f)$.

Theorem 3.3: Part III. If $\mathbf{f p t}_{\mathfrak{m}}(f)<\min \{1,|\boldsymbol{\delta}|\}$ and $p>\max \left\{d_{1}, \cdots, d_{n}\right\}$, then $\tau\left(\mathbf{f p t}_{\mathfrak{m}}(f) \bullet f\right)=\mathfrak{m}$.

Proof. Let $L=\max \left\{N: \sum_{i=1}^{n} \delta_{i}^{(e)} \leq p-1\right.$ for $\left.0 \leq e \leq N\right\}$, and set $\lambda:=\left|\langle\boldsymbol{\delta}\rangle_{L}\right|+\frac{1}{p^{L}}$. By definition, $\lambda^{(e)}=\sum_{i=1}^{n} \delta_{i}^{(e)}$ for $0 \leq e \leq L$ while $\lambda^{(e)}=p-1$ for $e \geq L+1$. As $\lambda<1$, there exists $1 \leq l \leq L$ such that $\lambda^{(l)}=\sum_{i=1}^{n} \delta_{i}^{(l)} \leq p-2$ and $\lambda^{(e)}=p-1$ for $e \geq l$. By Lemma 2.7 , our choice of $l$ guarantees that $\left(\begin{array}{c}p^{l}\left|\langle\boldsymbol{\delta}\rangle_{l}\right| \\ p^{l}\langle\boldsymbol{\delta}\rangle_{l}\end{array}\right) \neq 0 \bmod p$. As each $d_{i}$ is less than $p$, Corollary 5.4 and Lemma 1.7 combine to show that $\left(x_{1}, \cdots, x_{n}\right) \subseteq\left(f^{p^{l}\left|\langle\boldsymbol{\delta}\rangle_{l}\right|+1}\right)^{\left[\frac{1}{\left.p^{l}\right]}\right.}=\boldsymbol{\tau}(\lambda \bullet f)$.

\section{On (higher) F-Jumping numbers of FERMAT HyPERSuRfaces}

Notation 6.1. We now assume $f$ is a $\mathbb{L}^{*}$-linear combination of $x_{1}^{d_{1}}, \cdots, x_{n}^{d_{n}}$, and write $f=u_{1} x_{1}^{d}+\cdots+u_{d} x_{d}^{d}$. We continue to use $\delta$ to denote $\frac{1}{d}$.

Remark 6.2. Supposes $p>d$, and fix integers $\omega \geq 1$ and $1 \leq a<d$ such that $p=d \cdot \omega+a$. Isolating $\delta=\frac{1}{d}$ in this equation shows that $\delta=\frac{\omega}{p}+\frac{a}{d} \cdot \frac{1}{p}=\frac{\omega}{p}+(a \delta) \cdot \frac{1}{p}$. From this, we conclude that $\delta^{(1)}=\omega$ and that $\delta^{(e+1)}=(a \delta)^{(e)}$ for all $e \geq 1$.

The following Lemma will be key in proving Corollary 3.5.

Lemma 6.3. Suppose that $p>d>2$ and $p \equiv a \bmod d$. If $a \geq 2$, then $(d-1) \cdot \delta^{(2)} \geq p+1$.

Proof. Suppose, by means of contradiction, that $(d-1) \cdot \delta^{(2)} \leq p$. As $p$ is prime and both $d-1$ and $\delta^{(2)}$ are less than $p$, equality cannot hold. In particular,

$$
(d-1) \cdot \delta^{(2)} \leq p-1
$$

By Remark 6.2, we know $\frac{a}{d}=\sum_{e \geq 1} \frac{\delta^{(e+1)}}{p^{e}}$, and combining this observation with (8) shows

$$
\begin{aligned}
(d-1) \cdot \frac{a}{d}=\frac{(d-1) \cdot \delta^{(2)}}{p}+(d-1) \cdot \sum_{e=2}^{\infty} \frac{\delta^{(e+1)}}{p^{e}} & \leq \frac{(d-1) \cdot \delta^{(2)}}{p}+\frac{d-1}{p} \\
& \leq \frac{p-1}{p}+\frac{d-1}{p}=1+\frac{d-2}{p} .
\end{aligned}
$$

However, as $a \geq 2,(d-1) \cdot \frac{a}{d} \geq(d-1) \cdot \frac{2}{d}=1+\frac{d-2}{d}$, and comparing this with (9) shows $\frac{d-2}{d} \leq \frac{d-2}{p}$, which implies that $p \leq d$, a contradiction.

Corollary 3.5. We have the following formula for $\mathbf{f p t}_{\mathfrak{m}}(f)$ :

$$
\mathbf{f p t}_{\mathfrak{m}}(f)= \begin{cases}\frac{1}{p^{l}} & \text { if } p^{l} \leq d<p^{l+1} \text { for some } l \geq 1 \\ 1-\frac{a-1}{p} & \text { if } 0<d<p \text { and } p \equiv a \bmod d \text { with } 1 \leq a<d\end{cases}
$$

Proof. If $p^{l} \leq d<p^{l+1}$, then $\frac{1}{p^{l+1}}<\delta \leq \frac{1}{p^{l}}$. Consequently, $\delta^{(e)}$ for $1 \leq e \leq l$ and $\delta^{(l+1)} \neq 0$. Adding $d$ copies of $\delta^{(l+1)}$ yields $d \cdot \delta^{(l+1)} \geq d \geq p^{l} \geq p$. In the notation of Theorem 3.1, we have that $L=l$, and as $\langle\delta\rangle_{l}=0, \mathbf{f p t}_{\mathfrak{m}}(f)=d \cdot\langle\delta\rangle_{l}+\frac{1}{p^{l}}=\frac{1}{p^{l}}$. 
We now assume that $p>d$. If $a=1$, the identities in Remark 6.2 imply $\delta^{(e)}=\omega$ for all $e \geq 1$. As $d \cdot \delta^{(e)}=d \cdot \omega=p-1$, it follows that $d$ copies of $\delta$ add without carrying. By Theorem 3.1, $\mathbf{f p t}_{\mathfrak{m}}(f)=1$. Suppose now that $a \geq 2$ (which automatically implies $d>2$ ). Note that $d \cdot \delta^{(1)}=d \cdot \omega=p-a$ while $d \cdot \delta^{(2)}>p$ by Lemma 6.3. By Theorem 3.1, $\mathbf{f p t}_{\mathfrak{m}}(f)=d \cdot\langle\delta\rangle_{1}+\frac{1}{p}=\frac{d \cdot \omega}{p}+\frac{1}{p}=\frac{p-a+1}{p}$.

In order to prove Theorem 3.6, we will need the following lemmas.

Lemma 6.4. If $d$ is not a $p^{\text {th }}$ power, then $x_{i} \in\left(f^{N}\right)^{\left[\frac{1}{p^{e}}\right]}$ if and only if $0 \preccurlyeq d \cdot \boldsymbol{k}-p^{e} \cdot \mathbf{v}_{i} \prec p^{e} \cdot \mathbf{1}_{d}$ and $\left(\begin{array}{l}N \\ \boldsymbol{k}\end{array}\right) \neq 0 \bmod p$ for some $\boldsymbol{k}$ with $|\boldsymbol{k}|=N$.

Proof. For every $\boldsymbol{k} \in \mathbb{N}^{d}$, there is a unique element $\mathbf{c}_{\boldsymbol{k}} \in \mathbb{N}^{d}$ such that $0 \preccurlyeq d \cdot \boldsymbol{k}-p^{e} \cdot \mathbf{c}_{\boldsymbol{k}} \prec p^{e} \cdot \mathbf{1}_{d}$. If we set $\mu_{\boldsymbol{k}}:=\boldsymbol{x}^{d \cdot \boldsymbol{k}-p^{e} \cdot \mathbf{c}_{\boldsymbol{k}}}$, it follows that $\mu_{k} \in \mathscr{B}_{e}$ and that $\boldsymbol{x}^{d \cdot \boldsymbol{k}}=\boldsymbol{x}^{p^{e} \cdot \mathbf{c}_{\boldsymbol{k}}} \mu_{\boldsymbol{k}}$. Thus,

$$
f^{N}=\sum_{|\boldsymbol{k}|=N}\left(\begin{array}{c}
N \\
k
\end{array}\right) \boldsymbol{u}^{\boldsymbol{k}} \boldsymbol{x}^{d \cdot \boldsymbol{k}}=\sum_{|\boldsymbol{k}|=N}\left(\left(\left(\begin{array}{l}
N \\
\boldsymbol{k}
\end{array}\right) \boldsymbol{u}^{k}\right)^{1 / p^{e}} \boldsymbol{x}^{\boldsymbol{c}_{\boldsymbol{k}}}\right)^{p^{e}} \mu_{\boldsymbol{k}} .
$$

Let $I$ denote the ideal generated by the elements $\left(\left(\begin{array}{l}N \\ \boldsymbol{k}\end{array}\right) \boldsymbol{u}^{\boldsymbol{k}}\right)^{1 / p^{e}} \boldsymbol{x}^{\mathbf{c}_{k}}$. Apparently, (10) shows that $f^{N}$ is in $I^{\left[p^{e}\right]}$, and applying Lemma 1.4 then shows $\left(f^{N}\right)^{\left[\frac{1}{p^{e}}\right]} \subseteq I$. If $x_{i} \in\left(f^{N}\right)^{\left[\frac{1}{p^{e}}\right]}$, then $x_{i} \in I$, and so $x_{i}$ must be a unit multiple of one of the monomial generators of $I$. We conclude that $x_{i}=\boldsymbol{x}^{\mathbf{c}_{\boldsymbol{k}}}$ for some $\boldsymbol{k}$ with $\left(\begin{array}{l}N \\ \boldsymbol{k}\end{array}\right) \neq 0 \bmod p$.

Next, suppose that $0 \preccurlyeq d \cdot \boldsymbol{k}-p^{e} \cdot \mathbf{v}_{i} \prec p^{e} \cdot \mathbf{1}_{d}$ and $\left(\begin{array}{l}N \\ \boldsymbol{k}\end{array}\right) \neq 0 \bmod p$ for some $\boldsymbol{k}$ with $|\boldsymbol{k}|=N$, so that $\boldsymbol{x}^{d \cdot \boldsymbol{k}}=x_{i}^{p^{e}} \mu_{\boldsymbol{k}}$ is a supporting monomial of $f^{N}$. To show that $x_{i} \in\left(f^{N}\right)^{\left[\frac{1}{p^{e}}\right]}$, it suffices to show that $\boldsymbol{x}^{d \cdot \boldsymbol{k}}$ is the only supporting monomial of $f^{N}$ that is an $R^{p^{e}}$-multiple of $\mu_{\boldsymbol{k}}$. Let $\boldsymbol{x}^{d \cdot \boldsymbol{\kappa}}$ be another such monomial, so that $\boldsymbol{x}^{d \cdot \boldsymbol{\kappa}}=\boldsymbol{x}^{p^{e} \mathbf{c}} \mu_{\boldsymbol{k}}$ and $\boldsymbol{x}^{d \cdot \boldsymbol{k}}=x_{i}^{p^{e}} \mu_{\boldsymbol{k}}$. Solving for $\mu_{\boldsymbol{k}}$ in these expressions shows $\mu_{\boldsymbol{k}}=\boldsymbol{x}^{d \cdot \boldsymbol{k}-p^{e} \mathbf{c}}=\boldsymbol{x}^{d \cdot \boldsymbol{\kappa}-p^{e} \mathbf{v}_{i}}$, and so

$$
d \cdot(\boldsymbol{k}-\boldsymbol{\kappa})=p^{e} \cdot\left(\mathbf{c}-\mathbf{v}_{i}\right) .
$$

As $|\boldsymbol{k}|=|\boldsymbol{\kappa}|=N$, it follows from (11) that $|\mathbf{c}|=\left|\mathbf{v}_{i}\right|=1$, so that $\mathbf{c}=\mathbf{v}_{j}$ for some $j$. If $j \neq i$, then (11) shows that $d\left(k_{j}-\kappa_{j}\right)=p^{e}$, which contradicts the assumption that $d$ is not a $p^{\text {th }}$ power. Thus, $\mathbf{c}=\mathbf{v}_{i}$, and so $\boldsymbol{k}=\boldsymbol{\kappa}$ by (11).

Notation 6.5. From now on, we assume $p=d \cdot \omega+a$ for some $\omega \geq 0$ and $1 \leq a<d$.

Lemma 6.6. We have the following inequalities:

(1) $p<d(2 \omega+\lceil 2 a \delta\rceil-1)<2 p$.

(2) If $p<a(d-1)$, then $(d+1) \cdot \omega+\lceil 2 a \delta\rceil \leq p$.

(3) If $p>a(d-1)$, then $p<d(\omega+a-1)<2 p$.

Proof. The first point follows by applying the inequality $2 a \delta \leq\lceil 2 a \delta\rceil<2 a \delta+1$ and the identity $p=d \cdot \omega+a$. For the second point, note that $p=d \cdot \omega+a<a d-a$ by hypothesis, and it follows that $\omega+2 a \delta<a$. Adding $d \cdot \omega$ to both sides yields $(d+1) \cdot \omega+2 a \delta<a+d \cdot \omega=p$. The proof of the third point is similar, and is left to the reader.

Theorem 3.6: Part I. If $a \geq 2$ and $p<a(d-1)$, then

(1) $\boldsymbol{\tau}\left(\left(\frac{(d+1) \cdot \omega+\lceil 2 a \delta\rceil-1}{p}+\frac{p-1}{p^{2}}+\cdots+\frac{p-1}{p^{e}}\right) \bullet f\right)=\mathfrak{m}$ for all $e \geq 1$, and 
(2) $\boldsymbol{\tau}\left(\left(\frac{(d+1) \cdot \omega+\lceil 2 a \delta\rceil}{p}\right) \bullet f\right) \neq \mathfrak{m}$.

In particular, $\frac{(d+1) \cdot \omega+\lceil 2 a \delta\rceil}{p} \in(0,1]$ is an $F$-jumping number of $f$.

Proof. By Lemma 6.6, $\frac{(d+1) \cdot \omega+\lceil 2 a \delta\rceil}{p} \in(0,1]$. By Lemma 6.3, $(d-1) \cdot \delta^{(2)} \geq p+1$. Thus, there exists non-negative integers $l_{1}, \cdots, l_{d-1}$ such that $\sum l_{i}=p-1$ and $l_{i} \leq \delta^{(2)}$ for $1 \leq i \leq d-1$, with the inequality being strict for at least one $i$, which we are free to choose. In what follows, we assume that $l_{d-1}<\delta^{(2)}$. Fix $e \geq 3$, and set

$$
\boldsymbol{\lambda}_{e}:=\left(\frac{\omega}{p}+\frac{l_{1}}{p^{2}}, \cdots, \frac{\omega}{p}+\frac{l_{d-2}}{p^{2}}, \frac{\omega}{p}+\frac{l_{d-1}}{p^{2}}+\frac{p-1}{p^{3}}+\cdots+\frac{p-1}{p^{e}}, \frac{2 \omega+\lceil 2 a \delta\rceil-1}{p}\right) .
$$

By Remark $6.2, \delta^{(1)}=\omega$, and as $l_{d-1}<\delta^{(2)}$, the first $d-1$ entries of $\boldsymbol{\lambda}_{e}$ are less than or equal to $\langle\delta\rangle_{2}$. Set $\boldsymbol{k}:=p^{e} \boldsymbol{\lambda}_{e}$. It follows that the first $d-1$ entries of $d \cdot \boldsymbol{k}$ are less than or equal to $d \cdot p^{e}\langle\delta\rangle_{2}$, and thus strictly less than $p^{e}$ while, by Lemma 6.6, the last entry of $d \cdot \boldsymbol{k}$ is strictly between $p^{e}$ and $2 p^{e}$. Thus, $\mathbf{0} \preccurlyeq d \cdot \boldsymbol{k}-p^{e} \cdot \mathbf{v}_{d} \prec p^{e} \cdot \mathbf{1}_{d}$. By construction, the entries of $\boldsymbol{k}$ add without carrying (in base $p$ ), so $\left(\begin{array}{c}|\boldsymbol{k}| \\ \boldsymbol{k}\end{array}\right) \neq 0 \bmod p$. Finally,

$$
\begin{aligned}
|\boldsymbol{k}| & =p^{e} \cdot\left(\frac{(d+1) \cdot \omega+\lceil 2 a \delta\rceil-1}{p}+\sum_{i=1}^{d-1} \frac{l_{i}}{p^{2}}+\frac{p-1}{p^{3}}+\cdots+\frac{p-1}{p^{e}}\right) \\
& =p^{e} \cdot\left(\frac{(d+1) \cdot \omega+\lceil 2 a \delta\rceil-1}{p}+\frac{p-1}{p^{2}}+\frac{p-1}{p^{3}} \cdots+\frac{p-1}{p^{e}}\right) .
\end{aligned}
$$

We then apply Lemmas 6.4 and 1.7 to deduce that

$$
x_{d} \in\left(f^{|\boldsymbol{k}|}\right)^{\left[\frac{1}{p^{e}}\right]}=\boldsymbol{\tau}\left(\left(\frac{(d+1) \cdot \omega+\lceil 2 a \delta\rceil-1}{p}+\frac{p-1}{p^{2}}+\cdots+\frac{p-1}{p^{e}}\right) \bullet f\right) .
$$

As this argument is symmetric in the variables, the first claim follows.

We now show that $\boldsymbol{\tau}\left(\frac{(d+1) \cdot \omega+\lceil 2 a \delta\rceil}{p} \bullet f\right) \neq \mathfrak{m}$. By way of contradiction, suppose that $x_{1}$ is in $\boldsymbol{\tau}\left(\frac{(d+1) \cdot \omega+\lceil 2 a \delta\rceil}{p} \bullet f\right)=\left(f^{(d+1) \cdot \omega+\lceil 2 a \delta\rceil}\right)^{\left[\frac{1}{p}\right]}$. By Lemma 6.4, there exists $\boldsymbol{k} \in \mathbb{N}^{d}$ with $|\boldsymbol{k}|=(d+1) \cdot \omega+\lceil 2 a \delta\rceil$ such that $0 \preccurlyeq d \cdot \boldsymbol{k}-p \cdot \mathbf{v}_{1} \prec p \cdot \mathbf{1}_{d}$. Restated, $0 \preccurlyeq \frac{1}{p} \cdot \boldsymbol{k}-\delta \cdot \mathbf{v}_{1} \prec \delta \cdot \mathbf{1}_{d}$, and applying Lemma 2.4 shows $\frac{1}{p} \cdot k_{i} \leq\langle\delta\rangle_{1}=\frac{\delta^{(1)}}{p}=\frac{\omega}{p}$ for all $2 \leq i \leq d$. These same inequalities also show $\frac{1}{p} \cdot k_{1}<2 \delta$, and summing these bounds shows

$$
(d+1) \cdot \omega+\lceil 2 a \delta\rceil=|\boldsymbol{k}|<(d-1) \cdot \omega+2 p \delta .
$$

Gathering the multiples of $\omega$ and multiplying through by $d$ implies $2 d \cdot \omega+d \cdot\lceil 2 a \delta\rceil<2 p$, which this is impossible as $2 d \cdot \omega+d \cdot\lceil 2 a \delta\rceil \geq 2 d \cdot \omega+d \cdot 2 a \delta=2(d \cdot \omega+a)=2 p$. We conclude that $x_{1}$ (and by symmetry, no variable) is in $\boldsymbol{\tau}\left(\frac{(d+1) \cdot \omega+\lceil 2 a \delta\rceil}{p} \bullet f\right)$.

Theorem 3.6: Part II. If $a \geq 2$ and $p>a(d-1)$, then $\boldsymbol{\tau}\left(\left(\frac{p-1}{p}+\cdots+\frac{p-1}{p^{e}}\right) \bullet f\right)=\mathfrak{m}$ for all $e \geq 1$. In particular, the only $F$-jumping numbers of $f$ in $(0,1]$ are $\mathbf{f p t}_{\mathfrak{m}}(f)$ and 1 .

Proof. As in the proof of Theorem 3.6: Part I, Lemma 6.3 guarantees there exists nonnegative integers $l_{1}, \cdots, l_{d-1}$ such that $\sum_{i=1}^{d-1} l_{i}=p-1$ and $l_{i} \leq \delta^{(2)}$ for $1 \leq i \leq d-1$, with 
at least one inequality being strict. We again assume $l_{d-1}<\delta^{(2)}$. Fix $e \geq 3$, and let

$$
\lambda_{e}:=\left(\frac{\omega}{p}+\frac{l_{1}}{p^{2}}, \cdots, \frac{\omega}{p}+\frac{l_{d-2}}{p^{2}}, \frac{\omega}{p}+\frac{l_{d-1}}{p^{2}}+\frac{p-1}{p^{3}}+\cdots+\frac{p-1}{p^{e}}, \frac{\omega+a-1}{p}\right) .
$$

Set $\boldsymbol{k}=p^{e} \cdot \boldsymbol{\lambda}_{e}$. By construction, the entries of $\boldsymbol{k}$ add without carrying, so $\left(\begin{array}{c}|\boldsymbol{k}| \\ \boldsymbol{k}\end{array}\right) \neq 0 \bmod p$. As in the proof of Part I of Theorem 3.6, one may verify that $\mathbf{0} \preccurlyeq d \cdot \boldsymbol{k}-p^{e} \cdot \mathbf{v}_{d} \prec p^{e} \cdot \mathbf{1}_{d}$, and

$$
\begin{aligned}
|\boldsymbol{k}| & =p^{e} \cdot\left(\frac{d \cdot \omega+a-1}{p}+\sum_{i=1}^{d-1} \frac{l_{i}}{p^{2}}+\frac{p-1}{p^{3}}+\cdots+\frac{p-1}{p^{e}}\right) \\
& =p^{e} \cdot\left(\frac{p-1}{p}+\frac{p-1}{p^{2}}+\frac{p-1}{p^{3}} \cdots+\frac{p-1}{p^{e}}\right) .
\end{aligned}
$$

Once more, Lemmas 6.4 and 1.7 imply $x_{d} \in\left(f^{|\boldsymbol{k}|}\right)^{\left[\frac{1}{p^{e}}\right]}=\boldsymbol{\tau}\left(\left(\frac{p-1}{p}+\frac{p-1}{p^{2}}+\cdots+\frac{p-1}{p^{e}}\right) \bullet f\right)$.

By the symmetry of this argument, we conclude that $\mathfrak{m} \subseteq \boldsymbol{\tau}\left(\left(\frac{p-1}{p}+\cdots+\frac{p-1}{p^{e}}\right) \bullet f\right)$.

\section{REFERENCES}

[BMS08] Manuel Blickle, Mircea Mustaţă, and Karen E. Smith. Discreteness and rationality of Fthresholds. Michigan Math. J., 57:43-61, 2008. Special volume in honor of Melvin Hochster. 2

[BMS09] Manuel Blickle, Mircea Mustaţă, and Karen E. Smith. F-thresholds of hypersurfaces. Trans. Amer. Math. Soc., 361(12):6549-6565, 2009. 2

[Dic02] L.E. Dickson. Theorems on the residues of multinomial coefficients with respect to a prime modulus. Quarterly Journal of Pure and Applied Mathematics, 33:378-384, 1902. 3

[Her11] Daniel J. Hernández. F-purity versus log canonicity for polynomials. preprint, 2011. 6

[HH90] Melvin Hochster and Craig Huneke. Tight closure, invariant theory, and the Briançon-Skoda theorem. J. Amer. Math. Soc., 3(1):31-116, 1990. 1

[HY03] Nobuo Hara and Ken-Ichi Yoshida. A generalization of tight closure and multiplier ideals. Trans. Amer. Math. Soc., 355(8):3143-3174 (electronic), 2003. 1

[Luc78] Edouard Lucas. Theorie des Fonctions Numeriques Simplement Periodiques. Amer. J. Math., 1878. 3

[McD01] Moira A. McDermott. Test ideals in diagonal hypersurface rings. J. Algebra, 237(1):38-48, 2001. 1

[McD03] Moira A. McDermott. Test ideals in diagonal hypersurface rings. II. J. Algebra, 264(1):296-304, 2003. 1

[MTW05] Mircea Mustaţă, Shunsuke Takagi, and Kei-ichi Watanabe. F-thresholds and Bernstein-Sato polynomials. In European Congress of Mathematics, pages 341-364. Eur. Math. Soc., Zürich, 2005. 4

[MY09] Mircea Mustaţă and Ken-Ichi Yoshida. Test ideals vs. multiplier ideals. Nagoya Math. J., 193:111128, 2009. 5 